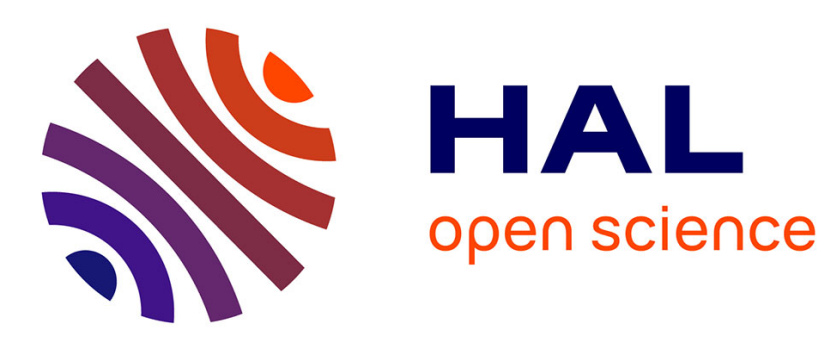

\title{
Pr4S3[AsS3]2: Praseodym(III)-Sulfid-Thioarsenat(III) mit Ketten und Schichten aus kondensierten [SPr4]-Tetraedern
}

Dong-Hee Kang, Thomas Schleid

\section{- To cite this version:}

Dong-Hee Kang, Thomas Schleid. Pr4S3[AsS3]2: Praseodym(III)-Sulfid-Thioarsenat(III) mit Ketten und Schichten aus kondensierten [SPr4]-Tetraedern. Journal of Inorganic and General Chemistry / Zeitschrift für anorganische und allgemeine Chemie, 2009, 635 (13-14), pp.2170. 10.1002/zaac.200900225 . hal-00512580

\section{HAL Id: hal-00512580 \\ https://hal.science/hal-00512580}

Submitted on 31 Aug 2010

HAL is a multi-disciplinary open access archive for the deposit and dissemination of scientific research documents, whether they are published or not. The documents may come from teaching and research institutions in France or abroad, or from public or private research centers.
L'archive ouverte pluridisciplinaire HAL, est destinée au dépôt et à la diffusion de documents scientifiques de niveau recherche, publiés ou non, émanant des établissements d'enseignement et de recherche français ou étrangers, des laboratoires publics ou privés. 
Pr4S3[AsS3]2: Praseodym(III)-Sulfid-Thioarsenat(III) mit Ketten und Schichten aus kondensierten [SPr4]-Tetraedern

\begin{tabular}{|r|l|}
\hline Journal: & Zeitschrift für Anorganische und Allgemeine Chemie \\
\hline Wanuscript ID: & zaac. 200900225.R1 \\
\hline Datey - Manuscript type: & Article \\
\hline Complete List of Authors: & $\begin{array}{l}\text { Kang, Dong-Hee; Universitaet Stuttgart, Institut fuer Anorganische } \\
\text { Chemie } \\
\text { Schleid, Thomas; Universitaet Stuttgart, Institut fuer Anorganische } \\
\text { Chemie }\end{array}$ \\
\hline Keywords: & Praseodymium, Sulfides, Thioarsenates(III), Crystal Structure \\
\hline
\end{tabular}

\section{\scholaroNE" \\ Manuscript Central}


Prof. Dr. Thomas Schleid, Institut für Anorganische Chemie, Universität Stuttgart, Pfaffenwaldring 55, D - 70569 Stuttgart

Pfaffenwaldring 55

D-70569 Stuttgart

An den Editor der Zeitschrift für

Anorganische und Allgemeine Chemie

Prof. Dr. Martin Jansen

Max-Planck-Institut für Festkörperforschung

Heisenbergstr. 1

70569 Stuttgart

3. Juli 2009

\begin{abstract}
Lieber Martin,
\end{abstract}
wir haben fast alle Anregungen der beiden Gutachter $\mathrm{zu}$ unserem Artikel mit dem Titel „Pr $\mathrm{Pr}_{4} \mathrm{~S}_{3}\left[\mathrm{AsS}_{3}\right]_{2}$ : Praseodym(III)-Sulfid-Thioarsenat(III) mit Ketten und Schichten aus kondensierten [SPr 4 ]-Tetraedern“ (Manuscript ID: zaac.200900225) in unsere Korrekturen einfließen lassen. Die zusätzlichen Literaturzitate (samt der noch zu erwähnenden Verbindungen) wurden eingebaut, die experimentellen Ergänzungen zum Auswaschen des Flussmittels wurden gegeben, die (äquivalenten isotropen) Auslenkungsparameter wurden durchgehend im Text und in den Tabellen als solche bezeichnet, auf die Tabelle mit den anisotropen Auslenkungsparametern wurde verzichtet, die offensichtlichen Druckfehler wurden beseitigt, die zahlreichen Striche an den Pr-Teilchen in der Abstands- und Winkeltabelle wurden entfernt und die Abbildungslegenden („Pr-S-Polyeder“ und „hexagonal dichteste Stabpackung“) wurden wie gewünscht verbessert. Auf den jeweils einen, stark aufgeweiteten Winkel in beiden [ $\left.\mathrm{SPr}_{4}\right]$-Tetraedern wurde insofern eingegangen, als ein neues Bild dazu kam, welches zeigt, dass lediglich die Pr-Pr-Kanten mit den großen Winkeln nicht von $\mathrm{S}^{2-}$-Anionen überspannt sind. Dies gilt übrigens auch für die Strukturen fast aller Sulfidhalogenide mit Ketten und/oder Schichten aus kantenverknüpften [ $\left.\mathrm{SM}_{4}\right]$-Tetraedern, über deren „herausragenden“ M-S-M-Singulärwinkel zwischen 130 und $140^{\circ}$ wir uns schon 
ZAAC

Page 2 of 26

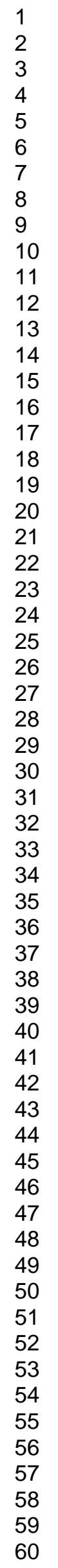

immer gewundert haben. Nun wissen wir Bescheid und haben auch noch etwas dazugelernt (so muss eine Reviewer-Bemerkung sich auswirken...)! Den Vergleich der Struktur von $\mathrm{Pr}_{4} \mathrm{~S}_{3}\left[\mathrm{AsS}_{3}\right]_{2}$ mit dem formelgleichen $\mathrm{La}_{4} \mathrm{O}_{3}\left[\mathrm{AsS}_{3}\right]_{2}$ haben wir trotz der betrüblichen ArsenFehlordnung beibehalten, denn uns kommt es ja auf die unterschiedlichen kationischen $\left\{\left[\mathrm{Ch}_{3} \mathrm{M}_{4}\right]^{6+}\right\}$-Teilstrukturen an, die davon unbetroffen sind. Auch unsere MAPLEAusführungen haben wir nicht groß verändert, denn hier liegt doch gerade der Reiz im Vergleich zwischen eher kovalenten und nicht ganz so kovalenten, also ionischen Verbindungen. Für das Tripel $\operatorname{Pr}_{4} \mathrm{As}_{2} \mathrm{~S}_{9}$ (also $\mathrm{Pr}_{4} \mathrm{~S}_{3}\left[\mathrm{AsS}_{3}\right]_{2}$ ) / $\mathrm{Pr}_{2} \mathrm{~S}_{3} / \mathrm{As}_{2} \mathrm{~S}_{3}$ sind die Resultate doch beeindruckend gut, wenn man „Vergleichbares mit Vergleichbarem vergleicht“ (Zitat Rudolf Hoppe, seines Zeichens Vater des MAPLE-Konzepts). Ich hoffe, dass wir durch unsere Verbesserungen ein qualitativ hochwertigeres Produkt abgeliefert haben und grüße Dich ganz herzlich von ,nebenan“,

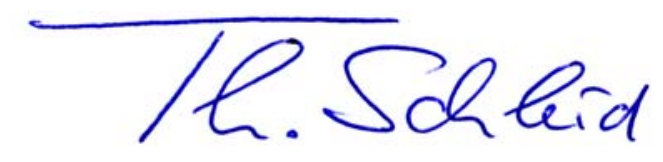

Dein Thomas

Wiley-VCH 
Z. Anorg. Allg. Chem.

\section{$\operatorname{Pr}_{4} \mathrm{~S}_{3}\left[\mathrm{AsS}_{3}\right]_{2}$ : Praseodym(III)-Sulfid-Thioarsenat(III) mit Ketten und Schichten aus kondensierten $\left[\mathrm{SPr}_{4}\right]$-Tetraedern}

\section{Dong-Hee Kang und Thomas Schleid*}

Stuttgart, Institut für Anorganische Chemie der Universität

Bei der Redaktion eingegangen am

2009.

\footnotetext{
* Prof. Dr. Thomas Schleid

Institut für Anorganische Chemie, Universität Stuttgart

Pfaffenwaldring 55

D-70569 Stuttgart

Telefax: $+49(0) 711 / 685-64241$

E-Mail: schleid@iac.uni-stuttgart.de
} 


\title{
$\operatorname{Pr}_{4} \mathrm{~S}_{3}\left[\mathrm{AsS}_{3}\right]_{2}$ : Praseodymium(III) Sulfide Thioarsenate(III) with Chains and Layers of Condensed [SPr 4 Tetrahedra
}

\begin{abstract}
Single crystals of $\operatorname{Pr}_{4} \mathrm{~S}_{3}\left[\mathrm{AsS}_{3}\right]_{2}$ can be obtained through the reaction between the elements praseodymium, arsenic and sulfur using $\mathrm{CsCl}$ as fluxing agent in torch-sealed evacuated silica ampoules. The starting materials were heated for ten days at $700{ }^{\circ} \mathrm{C}$ and then cooled down slowly to room temperature. The transparent, pale greenish yellow platelets crystallize orthorhombically $(a=$ 2910.6(2), $b=699.74(5), c=704.51(5) \mathrm{pm}$ ) in space group $P b c n$ (no. 60) with $\mathrm{Z}=4$. The new compound $\operatorname{Pr}_{4} \mathrm{As}_{2} \mathrm{~S}_{9}\left(\equiv \operatorname{Pr}_{4} \mathrm{~S}_{3}\left[\mathrm{AsS}_{3}\right]_{2}\right.$ ) contains two crystallographically independent $\operatorname{Pr}^{3+}$ cations. $(\operatorname{Pr} 1)^{3+}$ shows a bicapped trigonal prismatic environment of eight sulfur atoms $(\mathrm{CN}=8)$, whereas $(\operatorname{Pr} 2)^{3+}$ is surrounded by only seven sulfur atoms in the shape of a monocapped trigonal prism $(\mathrm{CN}=$ 7). The isolated pyramidal thioarsenate(III) anions $\left[\mathrm{AsS}_{3}\right]^{3-}$ are formed as $\psi^{1}$-tetrahedra by the three sulfur atoms $\left(\mathrm{d}\left(\mathrm{As}^{3+}-\mathrm{S}^{2-}\right)=223-228 \mathrm{pm}, \Varangle(\mathrm{S}-\mathrm{As}-\mathrm{S})=94-104^{\circ}\right)$ and a non-binding electron pair (lone pair) at each $\mathrm{As}^{3+}$ cation. Two of the five crystallographically different sulfur atoms (S1 and S2) are not connected to $\mathrm{As}^{3+}$ at all, but coordinated by four $\operatorname{Pr}^{3+}$ cations each to build up slightly distorted $\left[\mathrm{SPr}_{4}\right]^{10+}$ tetrahedra $\left(\mathrm{d}\left(\mathrm{S}^{2-}-\mathrm{Pr}^{3+}\right)=281-289 \mathrm{pm}, \Varangle(\mathrm{Pr}-\mathrm{S}-\mathrm{Pr})=98-146^{\circ}\right)$. Thus the crystal structure of $\operatorname{Pr}_{4} \mathrm{~S}_{3}\left[\mathrm{AsS}_{3}\right]_{2}$ exhibits endless ${ }_{\infty}^{1}\left\{\left[(\mathrm{~S} 1) \mathrm{Pr}_{4 / 2}\right]^{4+}\right\}$ chains and ${ }_{\infty}^{2}\left\{\left[(\mathrm{~S} 2) \mathrm{Pr}_{4 / 4}\right]^{+}\right\}$layers, which consist of trans-edge condensed $\left[(\mathrm{S} 1) \mathrm{Pr}_{4}\right]^{10+}$ tetrahedra for the chains and $\left[(\mathrm{S} 2) \mathrm{Pr}_{4}\right]^{10+}$ tetrahedra sharing four common edges for layers. The lone pairs at the $\mathrm{As}^{3+}$ cations close around the ${ }_{\infty}^{1}\left\{\left[(\mathrm{~S} 1) \mathrm{Pr}_{4 / 2}\right]^{4+}\right\}$ chains running along [001]. The ${ }_{\infty}^{2}\left\{\left[(\mathrm{~S} 2) \mathrm{Pr}_{4 / 4}\right]^{+}\right\}$layers spread out parallel to the (100) plane and complete the crystal structure of $\operatorname{Pr}_{4} \mathrm{~S}_{3}\left[\mathrm{AsS}_{3}\right]_{2}$ according to ${ }_{\infty}^{1}\left\{\left[(\mathrm{~S} 1)(\operatorname{Pr} 1)_{4 / 2}\right]^{4+}\right\}_{\infty}^{2}\left\{\left(\left[(\mathrm{~S} 2)(\mathrm{Pr} 2)_{4 / 4}\right]^{+}\right)_{2}\right\}$ ${ }_{\infty}^{0}\left\{\left([\mathrm{As}(\mathrm{S} 3)(\mathrm{S} 4)(\mathrm{S} 5)]^{3-}\right)_{2}\right\}$.
\end{abstract}

Keywords: Praseodymium; Sulfides; Thioarsenates(III); Crystal Structure 


\section{Einleitung}

Arsenatome weisen in Verbindungen mit elektronegativeren Elementen wie etwa den Chalkogenen (Sauerstoff oder Schwefel) meist die Oxidationszahlen $+\mathrm{III}$ oder $+\mathrm{V}$ auf. In komplexen Anionen mit Sauerstoff, den Oxoarsenat-Anionen, nimmt Arsen bevorzugt die Oxidationsstufe $+\mathrm{V}$ ein und tritt dann in Form von $\left[\mathrm{AsO}_{4}\right]^{3-}$-Tetraedern (z. B. $\mathrm{A}_{3}\left[\mathrm{AsO}_{4}\right], \mathrm{A}=$ Alkali-Metall [1]; $\mathrm{E}_{3}\left[\mathrm{AsO}_{4}\right]_{2}, \mathrm{E}=$ Erdalkali-Metall [2]; $\mathrm{M}\left[\mathrm{AsO}_{4}\right.$ ], $\mathrm{M}=$ Selten-Erd-Metall [3]) strukturell in Erscheinung. Seltener tritt bei Lanthanid-Verbindungen die Oxidationsstufe $+\mathrm{III}$ in $\psi^{1}$-tetraedrischen $\left[\mathrm{AsO}_{3}\right]^{3-}$-Anionen (z. B. isoliert im Formeltyp $\mathrm{M}_{3} \mathrm{OCl}\left[\mathrm{AsO}_{3}\right]_{2}, \mathrm{M}=\mathrm{La}$ und $\mathrm{Gd}$ [4], eckenverknüpft im Formeltyp $\mathrm{M}_{4}\left[\mathrm{As}_{2} \mathrm{O}_{5}\right]_{2}\left[\mathrm{As}_{4} \mathrm{O}_{8}\right], \mathrm{M}=\mathrm{Nd}$ und Sm [5]) auf. Im Gegensatz zu der dominanten Oxidationsstufe $+\mathrm{V}$ bei den Oxoarsenat-Anionen weisen die komplexen Anionen des Arsens mit Schwefel, die Thioarsenat-Anionen, gerade den gegenteiligen Trend auf. Die Kristallstrukturen von ternären Alkalimetall-Thioarsenaten(III) der Zusammensetzungen $\mathrm{A}_{3}\left[\mathrm{AsS}_{3}\right](\mathrm{A}=\mathrm{Li}$ und $\mathrm{Na})$ [6], $\mathrm{NaAsS}_{2}$ [7] und $\mathrm{Cs}_{2} \mathrm{As}_{8} \mathrm{~S}_{13}$ [8], die isolierte oder eckenverknüpfte $\psi^{1}$-tetraedrische Thioarsenat(III)-Anionen $\left[\mathrm{AsS}_{3}\right]^{3-}$ mit stereochemisch aktivem, nicht-bindendem Elektronenpaar (lone pair) enthalten, sind schon lange bekannt. Dagegen trifft man das Thioarsenat(V)-Anion nur in den Verbindungen der Zusammensetzung $\mathrm{M}_{3}\left[\mathrm{AsS}_{4}\right](\mathrm{M}=\mathrm{K}, \mathrm{Cu}, \mathrm{Ag}$ und $\mathrm{Tl})[9,10]$ an. In letzter Zeit wurden darüber hinaus aber auch quaternäre Alkalimetall-Thioarsenate(III/V) mit den unterschiedlichsten Metallen und Formeltypen wie etwa $\mathrm{KCu}_{2} \mathrm{AsS}_{3}$ und $\mathrm{KCu}_{4} \mathrm{AsS}_{4}$ [11], $\mathrm{KAg}_{2} \mathrm{AsS}_{4}$ [12], $\mathrm{Cs}_{2} \mathrm{AgAsS}_{4}$ [13], $\mathrm{K}_{2} \mathrm{AuAsS}_{4}[14], \mathrm{KSnAsS}_{5}$ und $\mathrm{K}_{2} \mathrm{SnAs}_{2} \mathrm{~S}_{6}$ [15], $\mathrm{Cs}_{2} \mathrm{SnAs}_{2} \mathrm{~S}_{9}$ [16] sowie $\mathrm{A}_{8} \mathrm{Mn}_{2} \mathrm{As}_{4} \mathrm{~S}_{16}(\mathrm{~A}=\mathrm{K}, \mathrm{Rb}$ und Cs) [17] beschrieben. Überraschenderweise sind jedoch die ternären Thioarsenate(III/V) mit den dreiwertigen Selten-Erd-Metallen der einfachsten Zusammensetzungen M[AsS 3 ] und M[AsS $\left.\mathrm{A}_{4}\right]$ überhaupt noch nicht bekannt. Nur das ternäre Europium(II)-Thioarsenat(V) $\mathrm{Eu}_{3}\left[\mathrm{AsS}_{4}\right]_{2}$ [18], die quaternären Alkalimetall-Europium(II)-Thioarsenate(III) $\mathrm{A}_{\mathrm{x}} \mathrm{Eu}^{\mathrm{II}}{ }_{3-\mathrm{y}} \mathrm{As}^{\mathrm{III}}{ }_{5-\mathrm{z}} \mathrm{S}_{10}(\mathrm{~A}=\mathrm{Li}$ und Na) [18] sowie die Kalium-Lanthanoid(III)-Thioarsenate(V) $\mathrm{K}_{3} \mathrm{M}\left[\mathrm{AsS}_{4}\right]_{2}(\mathrm{M}=\mathrm{Nd}$, Sm und Gd) [19] wurden $\mathrm{zu}$ diesem Themenkreis bereits dokumentiert. Auch halogenidhaltige Arsen(III)-Sulfide der 
Zusammensetzungen $\mathrm{Cs}_{2} \mathrm{CeCl}_{2}$ [AsS $\mathrm{As}_{3}$ [20], $\mathrm{M}_{3} \mathrm{~S}_{2} \mathrm{Cl}_{2}\left[\mathrm{AsS}_{3}\right.$ ( $\mathrm{M}=\mathrm{La}$ und Pr) [21] und $\mathrm{La}_{2}\left(\mathrm{~S}_{2}\right) \mathrm{X}\left[\mathrm{AsS} \mathrm{S}_{3}\right.$ ] $(\mathrm{X}=\mathrm{Cl}$ und $\mathrm{Br}$ ) [22] konnten vor kurzem dargestellt und strukturell charakterisiert werden, indem halogenidflussmittel-unterstützte Versuche zur Synthese der ersten M[AsS $\left.\mathrm{As}_{3}\right]$ - und M[AsS $\left.\mathrm{A}_{4}\right]$ Repräsentanten kläglich scheiterten.

\section{Experimentelles}

Das Praseodym(III)-Sulfid-Thioarsenat(III) der Zusammensetzung $\operatorname{Pr}_{4} \mathrm{As}_{2} \mathrm{~S}_{9}$, besser $\operatorname{Pr}_{4} \mathrm{~S}_{3}\left[\mathrm{AsS}_{3}\right]_{2}$, fiel als Nebenprodukt bei Versuchen zur Synthese des noch immer unbekannten einfachsten Praseodym(III)-Thioarsenats(III) $\operatorname{Pr}\left[\mathrm{AsS}_{3}\right]$ durch Umsetzung von metallischem Praseodym (Chempur; 99,9 \%), grauem Arsen (Heraeus; 99,9 \%) und elementarem Schwefel (Chempur; 99,9 \%) im molaren Verhältnis von $1: 1: 3$ mit Caesiumchlorid (Chempur; 99,9\%) als Flussmittel-Additiv für besseres Kristallwachstum an. Das Reaktionsgemenge wurde in evakuierten Kieselglasampullen für zehn Tage bei $700{ }^{\circ} \mathrm{C}$ erhitzt und lieferte nach langsamem Abkühlen mit $50{ }^{\circ} \mathrm{C}$ pro Tag auf Raumtemperatur transparente, blass grünlich-gelbe, plättchenförmige Einkristalle der Titelverbindung. Aufgrund ihrer relativen Instabilität gegenüber feuchter Luft und Wasser konnte sie allerdings nach Auswaschen des Flussmittels nicht phasenrein erhalten werden. Ein ausgewählter Kristall wurde mittels Röntgendiffraktometrie (Kappa-CCD, Fa. Bruker-Nonius) mit Mo-Ka-Strahlung vermessen und die Struktur mit Hilfe von Einkristalldiffraktometerdaten bei Raumtemperatur ermittelt. Weitere Einzelheiten zur Bestimmung und Verfeinerung der Kristallstruktur von $\operatorname{Pr}_{4} \mathrm{~S}_{3}\left[\mathrm{AsS}_{3}\right]_{2}$ sind in Tabelle 1 zusammengefasst. Die Tabelle 2 enthält die Atomkoordinaten und die Koeffizienten der äquivalenten isotropen Auslenkungsparameter. Tabelle 3 listet ausgewählte interatomare Abstände und Winkel auf und über die Motive der gegenseitigen Zuordnung informiert Tabelle 4. Zudem können weiterreichende Daten zur Kristallstrukturuntersuchung beim Fachinformationszentrum 
Karlsruhe (D-76344 Eggenstein-Leopoldshafen, E-Mail: crysdata@ fiz-karlsruhe.de) unter Angabe der Hinterlegungsnummer CSD-419663 für $\mathrm{Pr}_{4} \mathrm{As}_{2} \mathrm{~S}_{9}$ oder $\mathrm{Pr}_{4} \mathrm{~S}_{3}\left[\mathrm{AsS}_{3}\right]_{2}$ erfragt werden. 


\section{Ergebnisse und Diskussion}

Das ternäre Sulfid der Zusammensetzung $\mathrm{Pr}_{4} \mathrm{As}_{2} \mathrm{~S}_{9}$ kristallisiert orthorhombisch in der Raumgruppe Pbcn (Nr. 60) mit vier Formeleinheiten pro Elementarzelle. Wie schon aus der strukturierten Formel $\operatorname{Pr}_{4} \mathrm{~S}_{3}\left[\mathrm{AsS}_{3}\right]_{2}$ ersichtlich, finden sich in der Kristallstruktur isolierte $\psi^{1}$-tetraedrische Thioarsenat(III)Anionen $\left[\mathrm{AsS}_{3}\right]^{3-}$, die aus einem stereochemisch aktiven, nicht-bindenden Elektronenpaar (lone pair) und drei Schwefelatomen (S3, S4 und S5) am $\mathrm{As}^{3+}$-Kation bestehen. Die Abstände d(As $\left.{ }^{3+}-\mathrm{S}^{2-}\right)$ weisen Werte zwischen 223 und 228 pm auf, was sehr gut mit den vergleichbaren Abständen d(As ${ }^{3+}-$ $\left.\mathrm{S}^{2-}\right)=220-230 \mathrm{pm}$ im Mineral Orpiment des Arsensesquisulfids $\mathrm{As}_{2} \mathrm{~S}_{3}$ [23] übereinstimmt. Die Winkel liegen mit $\Varangle($ S-As-S $)=94-104^{\circ}$ ebenfalls im erwarteten Bereich, verglichen mit $\Varangle($ S-AsS) $=93-106^{\circ}$ in $\mathrm{As}_{2} \mathrm{~S}_{3}$ (Auripigment) [23], wobei die Auslenkung von $\mathrm{As}^{3+}$ aus der Dreiecksebene seiner drei $\mathrm{S}^{2-}$-Liganden $111 \mathrm{pm}$ (gegenüber $109 \mathrm{pm}$ in $\mathrm{As}_{2} \mathrm{~S}_{3}$ [23]) beträgt. Die Thioarsenat(III)Einheiten $\left[\mathrm{AsS}_{3}\right]^{3-}$ koordinieren sowohl einfach über Schwefelecken als auch chelatisierend über Schwefelkanten an die $\mathrm{Pr}^{3+}$-Kationen (Abb. 1). In der Kristallstruktur liegen zwei kristallographisch unterschiedliche $\operatorname{Pr}^{3+}$-Kationen vor, wobei beide die allgemeine Wyckoff-Lage $8 d$ (Symmetrie: 1) einnehmen. Das $(\operatorname{Pr} 1)^{3+}$-Kation wird von acht Schwefelatomen in Form eines doppelt überkappten trigonalen Prismas mit Abständen von 282 - 324 pm umgeben (Abb. 2, oben). Der längste $(\operatorname{Pr} 1)^{3+}-$ $\mathrm{S}^{2-}$-Bindungsabstand ist mit $324 \mathrm{pm}$ zwar etwas länger als gewöhnliche $\mathrm{Pr}^{3+}-\mathrm{S}^{2-}$-Abstände (vgl. etwa $\mathrm{d}\left(\operatorname{Pr}^{3+}-\mathrm{S}^{2-}\right)=283-313$ pm im A-Typ von $\left.\operatorname{Pr}_{2} \mathrm{~S}_{3}[24]\right)$, doch sind sogar koordinativ wirksame $\operatorname{Pr}^{3+}-$ $\mathrm{S}^{2-}$-Abstände von $339 \mathrm{pm}$ wie etwa im Oxidsulfid $\operatorname{Pr}_{10} \mathrm{OS}_{14}$ [25] schon nachgewiesen worden. Hier haben ECoN-Berechnungen (ECoN $\equiv$ Effective Coordination Number, nach Hoppe [26]) gezeigt, dass dieser Abstand von $339 \mathrm{pm}(\delta$-ECoN $=0,45)$ in $\operatorname{Pr}_{10} \mathrm{OS}_{14}$ einen deutlichen Koordinationsbeitrag leistet. Das $(\operatorname{Pr} 2)^{3+}$-Kation weist eine siebenfache Umgebung aus Schwefelatomen in Form eines einfach überkappten trigonalen Prismas auf, wobei deren Abstände zum Zentralkation $(\operatorname{Pr} 2)^{3+}$ im Bereich von 281 bis 299 pm liegen (Abb. 2, unten). Der achte Abstand eines Schwefelatoms zum $(\operatorname{Pr} 2)^{3+}$-Kation beträgt $482 \mathrm{pm}(\delta$-ECoN $=0,00)$, daher betätigt $(\operatorname{Pr} 2)^{3+}$ im Gegensatz zu $(\operatorname{Pr} 1)^{3+}$ nur 
eine Koordinationszahl von sieben. Die vier $\left[\mathrm{AsS}_{3}\right]^{3-}$-Anionen sind sowohl chelatisierend über gemeinsame Schwefelkanten als auch terminal über gemeinsame Schwefelecken an das $\left[(\operatorname{Pr} 1) S_{8}\right]-$ Polyeder geknüpft. Im Falle des $\left[(\operatorname{Pr} 2) \mathrm{S}_{7}\right]$-Polyeders koordiniert jeweils ein $\left[\mathrm{AsS}_{3}\right]^{3-}$-Anion über Kante sowie eines über Ecke. In der Titelverbindung existieren fünf kristallographisch unabhängige Schwefelatome, wovon drei (S3, S4 und S5) die zuvor beschriebene die Thioarsenat(III)-Einheit $\left[\mathrm{AsS}_{3}\right]^{3-}$ bilden, während zwei Schwefelatome (S1 und S2), im Gegensatz zu drei Schwefelatomen des $\left[\mathrm{AsS}_{3}\right]^{3-}$-Anions, nicht an $\mathrm{As}^{3+}$-Kationen, sondern nur an $\mathrm{Pr}^{3+}$-Kationen koordiniert sind. Das Schwefelatom $\mathrm{S} 1$ ist von vier $(\operatorname{Pr} 1)^{3+}$-Kationen leicht verzerrt tetraedrisch umgeben $\left(\mathrm{d}\left((\mathrm{S} 1)^{2-}-(\operatorname{Pr} 1)^{3+}\right)\right.$ $=282(2 \times)$ und $289(2 \times)$ pm; $\left.\Varangle(\operatorname{Pr} 1-S 1-\operatorname{Pr} 1)=99-139^{\circ}\right)$. Während sich fünf Winkel $\Varangle(\operatorname{Pr} 1-\mathrm{S} 1-$ Pr1) zwischen 99 und $105^{\circ}$ bewegen, liegt der sechste mit $139^{\circ}$ deutlich über dem TeteraederIdealwert von $109,5^{\circ}$. Dies kann durch die weitere Koordination der $\left[(\mathrm{S} 1)(\operatorname{Pr} 1)_{4}\right]^{10+}$-Einheiten durch Sulfid-Anionen erklärt werden, denn fünf $\operatorname{Pr} 1-\operatorname{Pr} 1-$ Tetraederkanten sind von $S^{2-}$-Anionen chelatisierend überspannt, wogegen die sechste frei von $\mathrm{S}^{2-}$-Anionen bleibt und wohl daher ein größerer Winkel auftritt (Abb. 3, oben). Ganz ähnliche Beobachtungen gelten auch für die Ketten aus kondensierten $\left[\mathrm{SM}_{4}\right]^{10+}$-Tetraedern in den Kristallstrukturen der Sulfidhalogenide $\mathrm{Sm}_{2} \mathrm{SCl}_{4}$ [27], $\mathrm{Dy}_{3} \mathrm{~S}_{2} \mathrm{Cl}_{5}$ [28], $\mathrm{Nd}_{4} \mathrm{~S}_{3} \mathrm{Br}_{6}$ [29] und $\mathrm{Pr}_{7} \mathrm{~S}_{6} \mathrm{Cl}_{9}$ [30]. Diese [(S1)(Pr1 $\left.)_{4}\right]^{10+}-$ Tetraeder sind untereinander durch trans-Kantenverknüpfung verbunden und bilden Endlosketten der Zusammensetzung ${ }_{\infty}^{1}\left\{\left[(\mathrm{~S} 1)(\operatorname{Pr} 1)_{4 / 2}\right]^{4+}\right\}$ (Abb. 4, oben) entlang [001] aus, wie sie als anti-SiS ${ }_{2}$-analoge ${ }_{\infty}^{1}\left\{\left[\mathrm{SSm}_{4 / 2}\right]^{4+}\right\}-$ Ketten auch in $\mathrm{Sm}_{2} \mathrm{SCl}_{4}$ [27] anzutreffen sind. In Abbildung 4 (unten) wird die hexagonale Stabpackung aus diesen Strängen in Richtung der c-Achse sichtbar. Das Schwefelatom S2 bildet ebenfalls verzerrte $\left[(\mathrm{S} 2)(\operatorname{Pr} 2)_{4}\right]^{10+}-$ Tetraeder $\left(\mathrm{d}\left((\mathrm{S} 2)^{2-}-(\operatorname{Pr} 2)^{3+}\right)=281-285 \mathrm{pm}, \Varangle(\operatorname{Pr} 2-\mathrm{S} 2-\operatorname{Pr} 2)=98\right.$ - $107^{\circ}$ sowie $146^{\circ}$ ), wobei der größte Winkel erneut mit der nicht vorhandenen Koordination der betroffenen $\mathrm{Pr}^{3+}$-Kationen durch Sulfid-Anionen korreliert (Abb. 3, unten). Auch hier finden sich Analogen $\mathrm{zu}$ den Schichten aus kondensierten $\left[\mathrm{SM}_{4}\right]^{10+}$-Tetraedern in den Kristallstrukturen der Sulfidhalogenide LaSCl [31], NdSBr [32] oder GdSI und DySI [33]. Diese [(S2)(Pr2) $]^{10+}-$ Tetraeder 
bilden durch Kondensation über vier verknüpfende Kanten ${ }_{\infty}^{2}\left\{\left[(\mathrm{~S} 2)(\operatorname{Pr} 2)_{4 / 4}\right]^{+}\right\}$-Schichten parallel zur $b c$-Ebene (Abb. 5). Solche schachbrettartigen ${ }_{\infty}^{2}\left\{\left[\mathrm{SM}_{4 / 4}\right]^{+}\right\}-$Schichten wurden ebenfalls bereits für die Sulfidchloride des Formeltyps MSCl (M = La - Pr [31] und Er [34]) vorgestellt. Es fällt auf, dass die Titelverbindung nicht nur ${ }_{\infty}^{1}\left\{\left[\mathrm{SM}_{4 / 2}\right]^{4+}\right\}$-Ketten oder ${ }_{\infty}^{2}\left\{\left[\mathrm{SM}_{4 / 4}\right]^{+}\right\}$-Schichten enthält, sondern überraschenderweise beide Arten (Abb. 6) gleichzeitig. Außerdem folgt daraus, dass $\operatorname{Pr}_{4} \mathrm{~S}_{3}\left[\mathrm{AsS}_{3}\right]_{2}$ nicht isotyp zum sauerstoffhaltigen Lanthan-Thioarsenat(III) der formelanalogen Zusammensetzung $\mathrm{La}_{4} \mathrm{O}_{3}\left[\mathrm{AsS}_{3}\right]_{2}$ (orthorhombisch, Ibam (Nr. 72), $a=1903,2(4), b=1205,1(3), c=585,2(5) \mathrm{pm}, \mathrm{Z}=4$ ) [35] kristallisiert. In der Kristallstruktur von $\mathrm{La}_{4} \mathrm{O}_{3}\left[\mathrm{AsS}_{3}\right]_{2}$ kommen zwar auch zwei kristallographisch unterschiedliche Sauerstoffatome $\mathrm{O} 1$ und $\mathrm{O} 2$, analog $\mathrm{zu}$ den unterschiedlichen Schwefelatomen S1 und S2 der Titelverbindung vor. Jedoch bilden die korrespondierenden $\left[\mathrm{OLa}_{4}\right]^{10+}$-Tetraeder durch Kantenverknüpfung im Gegensatz $\mathrm{zu} \quad \operatorname{Pr}_{4} \mathrm{~S}_{3}\left[\mathrm{AsS}_{3}\right]_{2}$ bandartige ${ }_{\infty}^{1}\left\{\left[\mathrm{O}_{3} \mathrm{La}_{4}\right]^{6+}\right\}$-Dreifachketten entlang der $c$-Achse aus (Abb. 7, oben), wie sie in schwefelanaloger Form bereits im Sulfidbromid des Formeltyps $\mathrm{Nd}_{4} \mathrm{~S}_{3} \mathrm{Br}_{6}$ [29] als ${ }_{\infty}^{1}\left\{\left[\mathrm{~S}_{3} \mathrm{Nd}_{4}\right]^{6+}\right\}$-Bänder identifiziert wurden (Abb. 7, unten). Während die lone pairs an den $\mathrm{As}^{3+}$-Kationen der $\left[\mathrm{AsS}_{3}\right]^{3-}$-Einheiten in $\operatorname{Pr}_{4} \mathrm{~S}_{3}\left[\mathrm{AsS}_{3}\right]_{2}$ in Richtung der ${ }_{\infty}^{1}\left\{\left[(\mathrm{~S} 1)(\operatorname{Pr} 1)_{4 / 2}\right]^{4+}\right\}$-Ketten zu liegen kommen, zeigen die lone pairs an den $\mathrm{As}^{3+}$-Kationen der $\left[\mathrm{AsS}_{3}\right]^{3-}$-Einheiten in $\mathrm{La}_{4} \mathrm{O}_{3}\left[\mathrm{AsS}_{3}\right]_{2}$ eher in Richtung der leeren Kanalmitten, die durch die Lanthan-Schwefel-Polyeder gebildet werden. Dieser zweite offensichtliche Unterschied zwischen den beiden Kristallstrukturen wird durch den Vergleich von Abbildung 6 für $\operatorname{Pr}_{4} \mathrm{~S}_{3}\left[\mathrm{AsS}_{3}\right]_{2}$ mit Abbildung 7 (oben) für $\mathrm{La}_{4} \mathrm{O}_{3}\left[\mathrm{AsS}_{3}\right]_{2}$ besonders deutlich.

Ein weiteres Indiz für die Qualität der Kristallstruktur von Praseodym(III)-Sulfid-Thioarsenat(III) $\operatorname{Pr}_{4} \mathrm{~S}_{3}\left[\mathrm{AsS}_{3}\right]_{2}$ ergibt sich durch die Betrachtung der Madelung-Anteile der Gitterenergie (MAPLE nach Hoppe [36, 37]). Demnach erzielt die ternäre Verbindung $\operatorname{Pr}_{4} \mathrm{As}_{2} \mathrm{~S}_{9}(\mathrm{CN}(\operatorname{Pr} 1)=8: \mathrm{d}(\operatorname{Pr} 1-\mathrm{S})=282-$ $324 \mathrm{pm}, \mathrm{CN}(\operatorname{Pr} 2)=7: \mathrm{d}(\operatorname{Pr} 2-\mathrm{S})=281-299 \mathrm{pm})$ mit $8883 \mathrm{kcal} / \mathrm{mol}$ bei einer nur geringen Abweichung von $+1,2 \%$ gegenüber der MAPLE-Summe ihrer binären Komponenten $\operatorname{Pr}_{2} \mathrm{~S}_{3}(\mathrm{~A}-\mathrm{Typ}$, $\mathrm{CN}(\operatorname{Pr} 1)=8: \mathrm{d}(\operatorname{Pr} 1-\mathrm{S})=282-312 \mathrm{pm}, \mathrm{CN}(\operatorname{Pr} 2)=7: \mathrm{d}(\operatorname{Pr} 2-\mathrm{S})=283-300 \mathrm{pm})[24] \mathrm{und}_{\mathrm{As}_{2} \mathrm{~S}_{3}}$ 
(Auripigment oder Orpiment, $\mathrm{CN}(\mathrm{As})=3: \mathrm{d}(\mathrm{As} 1-\mathrm{S})=227-229 \mathrm{pm}, \mathrm{d}(\mathrm{As} 2-\mathrm{S})=224-230 \mathrm{pm})$ [23] entsprechend $2 \times \operatorname{MAPLE}\left(\mathrm{A}-\mathrm{Pr}_{2} \mathrm{~S}_{3}\right)+\operatorname{MAPLE}\left(\mathrm{As}_{2} \mathrm{~S}_{3}\right)\left(\sum=5816+2964=8780 \mathrm{kcal} / \mathrm{mol}\right)$ einen leichten Energiezuwachs. Darüber hinaus verbessert sich die Abweichung sogar noch ein wenig, wenn man den analogen Vergleich mit $\operatorname{Pr}_{2} \mathrm{~S}_{3}$ im C-Typ $(\mathrm{CN}(\operatorname{Pr})=8: \mathrm{d}(\operatorname{Pr}-\mathrm{S})=286-307 \mathrm{pm})$ [38] gemäß $2 \times \operatorname{MAPLE}\left(\mathrm{C}-\mathrm{Pr}_{2} \mathrm{~S}_{3}\right)+\operatorname{MAPLE}\left(\mathrm{As}_{2} \mathrm{~S}_{3}\right)\left(\sum=5878+2964=8842 \mathrm{kcal} / \mathrm{mol} ; \Delta=+0,5 \%\right)$ vornimmt. Dies überrascht etwas, da acht- und siebenfach von $\mathrm{S}^{2-}$-Anionen koordinierte $\operatorname{Pr}^{3+}$ Kationen in $\operatorname{Pr}_{4} \mathrm{As}_{2} \mathrm{~S}_{9}$ mit ausschließlich achtfach von $\mathrm{S}^{2-}$-Anionen koordinierten in $\mathrm{C}_{-} \mathrm{Pr}_{2} \mathrm{~S}_{3}$ verglichen werden. Der MAPLE-exotherme Trend für die formale Bildung von $\operatorname{Pr}_{4} \mathrm{As}_{2} \mathrm{~S}_{9}$ (三 $\operatorname{Pr}_{4} \mathrm{~S}_{3}\left[\mathrm{AsS}_{3}\right]_{2}$ ) aus den Binärkomponenten $\operatorname{Pr}_{2} \mathrm{~S}_{3}$ und $\mathrm{As}_{2} \mathrm{~S}_{3}$ bleibt davon jedoch unbetroffen. Der Blick auf Tabelle 4 (Motive der gegenseitigen Zuordnung) zeigt, dass dabei die Gewinn-und-VerlustRechnung für die Trikationen $\operatorname{Pr}^{3+}$ und $\mathrm{As}^{3+}$ kostenneutral bleibt: Sowohl $\mathrm{CN}\left(\mathrm{Pr}^{3+}\right)=7$ und 8 in A$\operatorname{Pr}_{2} \mathrm{~S}_{3}$ als auch $\mathrm{CN}\left(\mathrm{As}^{3+}\right)=3$ in $\mathrm{As}_{2} \mathrm{~S}_{3}$ werden unverändert in die Kristallstruktur von $\operatorname{Pr}_{4} \mathrm{~S}_{3}\left[\mathrm{AsS}_{3}\right]_{2}$ übertragen. Wenn es einen Gewinner gibt, dann sind es wohl die $\mathrm{S}^{2-}$-Anionen $(\mathrm{CN}=2)$ in $\mathrm{As}_{2} \mathrm{~S}_{3}$ (Auripigment mit Schichtstruktur aus eckenverknüpften $\left[\mathrm{AsS}_{3}\right]^{3-}-\psi^{1}-$ Tetraedern), denen durch die hoch koordinierten $\mathrm{S}^{2-}$-Anionen $\left(\mathrm{CN}=5\right.$ ) in $\operatorname{Pr}_{2} \mathrm{~S}_{3}$ (A-Typ) eine Überkompensation zu einer gleichmäßigen Viererkoordination für alle am Aufbau von $\operatorname{Pr}_{4} \mathrm{~S}_{3}\left[\mathrm{AsS}_{3}\right]_{2}$ beteiligten Sulfid-Teilchen im ternären $\operatorname{Pr}_{4} \mathrm{As}_{2} \mathrm{~S}_{9}$ zuteil wird.

\footnotetext{
Wir danken Frau cand.-chem. Inga Loke für ihre präparative Hilfe, Herrn Dr. Falk Lissner für die Einkristallmessung und dem Land Baden-Württemberg (Stuttgart) für die finanzielle Unterstützung durch Personal- und Sachmittel.
} 
Tabelle 1 Kristallographische Daten für $\operatorname{Pr}_{4} \mathrm{~S}_{3}\left[\mathrm{AsS}_{3}\right]_{2}$ und ihre Bestimmung

\begin{tabular}{|c|c|}
\hline Kristallsystem & orthorhombisch \\
\hline Raumgruppe & Pbcn (Nr. 60) \\
\hline Gitterkonstanten, $a(\mathrm{pm})$ & $2910,6(2)$ \\
\hline$b(\mathrm{pm})$ & $699,74(5)$ \\
\hline$c(\mathrm{pm})$ & $704,51(5)$ \\
\hline Zahl der Formeleinheiten $(\mathrm{Z})$ & 4 \\
\hline molares Volumen, $\mathrm{V}_{\mathrm{m}}\left(\mathrm{cm}^{3} \cdot \mathrm{mol}^{-1}\right)$ & $216,0(8)$ \\
\hline berechnete Dichte, $\mathrm{D}_{\mathrm{x}}\left(\mathrm{g} \cdot \mathrm{cm}^{-3}\right)$ & 4,639 \\
\hline Messgerät & $\kappa-\mathrm{CCD}$ (Fa. Bruker-Nonius) \\
\hline verwendete Strahlung & Mo-K $\alpha$ (Graphitmonochromator: $\lambda=71,07 \mathrm{pm}$ ) \\
\hline $\mathrm{F}(000)$ & 1784 \\
\hline$\Theta_{\max }(\mathrm{grd})$ & 28,32 \\
\hline Messbereich & $-38 \leq h \leq 38,-9 \leq k \leq 9,-9 \leq l \leq 9$ \\
\hline gemessene Reflexe & 15631 \\
\hline davon symmetrieunabhängig & 1774 \\
\hline$\mu\left(\mathrm{mm}^{-1}\right)$ & 19,19 \\
\hline Absorptionskorrektur & Programm X-SHAPE bzw. HABITUS [39] \\
\hline $\mathrm{R}_{\text {int }} / \mathrm{R}_{\sigma}$ & $0,074 / 0,038$ \\
\hline Strukturlösung und -verfeinerung & Programmpaket SHELX-97 [40] \\
\hline Streufaktoren & International Tables, Vol. C [41] \\
\hline $\mathrm{R}_{1}\left(\right.$ mit $\left|\mathrm{F}_{\mathrm{o}}\right| \geq 4 \sigma\left(\mathrm{F}_{\mathrm{o}}\right)$ für 1504 Reflexe $)$ & 0,031 \\
\hline $\mathrm{R}_{1} / w \mathrm{R}_{2}$ (für alle 1774 Reflexe) & $0,043 / 0,066$ \\
\hline Goodness of Fit (GooF) & 1,049 \\
\hline Extinktion $(\mathrm{g})$ & $0,00044(6)$ \\
\hline Restelektronendichte $\left(\mathrm{e}^{-} \cdot 10^{-6} \mathrm{pm}^{-3}\right)$ & $\max : 1,56 / \min :-1,23$ \\
\hline
\end{tabular}


Tabelle 2 Atomkoordinaten und Koeffizienten der äquivalenten isotropen Auslenkungsparameter $\left(\mathrm{U}_{\mathrm{eq}} / \mathrm{pm}^{2}\right)$ für $\mathrm{Pr}_{4} \mathrm{~S}_{3}\left[\mathrm{AsS}_{3}\right]_{2}$

\begin{tabular}{ccllll}
\hline Atom & Wyckoff-Lage & $x / a$ & $y / b$ & $z / c$ & $\mathrm{U}_{\mathrm{eq}}$ \\
\hline Pr1 & $8 d$ & $0,44333(1)$ & $0,29522(5)$ & $0,04334(5)$ & $87(1)$ \\
Pr2 & $8 d$ & $0,29296(1)$ & $0,20484(5)$ & $0,32131(5)$ & $79(1)$ \\
S1 & $4 c$ & 0 & $0,0627(3)$ & $1 / 4$ & $88(5)$ \\
S2 & $8 d$ & $0,23536(5)$ & $0,0004(2)$ & $0,5734(2)$ & $97(3)$ \\
As & $8 d$ & $0,09575(2)$ & $0,30503(9)$ & $0,07536(9)$ & $92(2)$ \\
S3 & $8 d$ & $0,45920(5)$ & $0,0834(2)$ & $0,3842(2)$ & $101(3)$ \\
S4 & $8 d$ & $0,35627(5)$ & $0,0545(2)$ & $0,0217(2)$ & $100(3)$ \\
S5 & $8 d$ & $0,38003(5)$ & $0,4256(2)$ & $0,3704(2)$ & $97(3)$ \\
\hline
\end{tabular}


Tabelle 3 Ausgewählte interatomare Abstände (d/pm) und Winkel ( $\Varangle /$ grd) für $\operatorname{Pr}_{4} \mathrm{~S}_{3}\left[\mathrm{AsS}_{3}\right]_{2}$

\begin{tabular}{|c|c|c|c|}
\hline \multicolumn{2}{|c|}{$\left[(\operatorname{Prl}) S_{8}\right]$-Polyeder: } & \multicolumn{2}{|c|}{$\left[(\operatorname{Pr} 2) S_{7}\right]$-Polyeder: } \\
\hline $\operatorname{Pr} 1-\mathrm{S} 1$ & 282,5 & $\operatorname{Pr} 2-S 2$ & 281,5 \\
\hline$-\mathrm{S} 3$ & 286,0 & $-\mathrm{S} 2{ }^{\prime}$ & 282,6 \\
\hline$-\mathrm{S} 1^{\prime}$ & 288,9 & $-\mathrm{S} 2 "$ & 283,0 \\
\hline$-\mathrm{S} 33^{\prime}$ & 291,4 & $-\mathrm{S} 22^{\prime \prime}$ & 284,8 \\
\hline$-\mathrm{S} 5$ & 294,9 & $-\mathrm{S} 4$ & 294,6 \\
\hline$-\mathrm{S} 4$ & 304,7 & $-\mathrm{S} 5$ & 298,8 \\
\hline$-\mathrm{S} 5^{\prime}$ & 308,8 & $-\mathrm{S} 44^{\prime}$ & 299,3 \\
\hline$-\mathrm{S} 3 "$ & 324,1 & & \\
\hline \multicolumn{4}{|c|}{$\left[A s S_{3}\right]^{3-}$-Anion: } \\
\hline As $-\mathrm{S} 3$ & 223,2 & S3-As-S4 & 94,1 \\
\hline$-\mathrm{S} 4$ & 226,7 & S3-As-S5 & 95,0 \\
\hline$-\mathrm{S} 5$ & 227,7 & S4-As-S5 & 104,4 \\
\hline \multicolumn{4}{|c|}{$\left[(\mathrm{S} 1)(\mathrm{Prl})_{4}\right]^{10+}-$ Tetraeder: } \\
\hline $\mathbf{S} 1-\operatorname{Pr} 1(2 \times)$ & 282,5 & $\operatorname{Pr} 1-\mathrm{S} 1-\operatorname{Pr} 1$ & 99,2 \\
\hline \multirow[t]{5}{*}{$-\operatorname{Pr} 1^{\prime}(2 \times)$} & 288,9 & & 101,1 \\
\hline & & & 101,1 \\
\hline & & & 105,3 \\
\hline & & & 105,3 \\
\hline & & & 138,8 \\
\hline \multicolumn{4}{|c|}{$\left[(\mathrm{S} 2)(\operatorname{Pr} 2)_{4}\right]^{10+}-$ Tetraeder: } \\
\hline $\mathbf{S} 2-\operatorname{Pr} 2$ & 281,5 & $\operatorname{Pr} 2-\mathrm{S} 2-\operatorname{Pr} 2$ & 98,5 \\
\hline$-\operatorname{Pr} 2^{\prime}$ & 282,6 & & 99,4 \\
\hline - Pr2" & 283,0 & & 100,9 \\
\hline \multirow[t]{3}{*}{ - Pr2"' } & 284,8 & & 101,1 \\
\hline & & & 107,1 \\
\hline & & & 146,2 \\
\hline
\end{tabular}


Tabelle 4 Motive der gegenseitigen Zuordnung in den Kristallstrukturen von $\operatorname{Pr}_{4} \mathrm{~S}_{3}\left[\mathrm{AsS}_{3}\right]_{2}$ (oben), $\mathrm{Pr}_{2} \mathrm{~S}_{3}$ (A-Typ, Mitte) und $\mathrm{As}_{2} \mathrm{~S}_{3}$ (Auripigment-Typ, unten)

\begin{tabular}{l|ccccc:c}
\hline & $\mathrm{S} 1$ & $\mathrm{~S} 2$ & $\mathrm{~S} 3$ & $\mathrm{~S} 4$ & $\mathrm{~S} 5$ & $\mathrm{CN}$ \\
\hline $\operatorname{Pr} 1$ & $2 / 4$ & $0 / 0$ & $3 / 3$ & $1 / 1$ & $2 / 2$ & 8 \\
$\operatorname{Pr} 2$ & $0 / 0$ & $4 / 4$ & $0 / 0$ & $2 / 2$ & $1 / 1$ & 7 \\
As & $0 / 0$ & $0 / 0$ & $1 / 1$ & $1 / 1$ & $1 / 1$ & 3 \\
\hdashline $\mathrm{CN}$ & 4 & 4 & 4 & 4 & 4 & \\
\hline
\end{tabular}

\begin{tabular}{l|ccc:c}
\hline & S1 & S2 & S3 & CN \\
\hline Pr1 & $2 / 2$ & $2 / 2$ & $4 / 4$ & 8 \\
$\operatorname{Pr} 2$ & $3 / 3$ & $3 / 3$ & $1 / 1$ & 7 \\
\hdashline $\mathrm{CN}$ & 5 & 5 & 5 & \\
\hline
\end{tabular}

\begin{tabular}{l|ccc:c}
\hline & $\mathrm{S} 1$ & $\mathrm{~S} 2$ & $\mathrm{~S} 3$ & $\mathrm{CN}$ \\
\hline As1 & $1 / 1$ & $1 / 1$ & $1 / 1$ & 3 \\
As2 & $1 / 1$ & $1 / 1$ & $1 / 1$ & 3 \\
\hdashline $\mathrm{CN}$ & 2 & 2 & 2 & \\
\hline
\end{tabular}




\section{Literatur}

[1] I. Abrahams, P. G. Bruce, W. I. F. David, A. R. West, J. Solid State Chem. 1994, 110, 243;

F. Emmerling, M. Idilbi, C. Röhr, Z. Naturforsch. 2002, 57 b, 599.

[2] R. Gopal, C. Calvo, Can. J. Chem. 1971, 49, 1036; N. Krishnamachari, C. Calvo, Acta Crystallogr. 1973, B 29, 2611; K. Bluhm, C. -H. Park, Z. Naturforsch. 1996, 51 b, 722.

[3] M. Schmidt, U. Müller, R. Cardoso Gil, E. Milke, M. Binnewies, Z. Anorg. Allg. Chem. 2005, 631, 1154; D.-H. Kang, P. Höss, Th. Schleid, Acta Crystallogr. 2005, E 61, i270; D.-H. Kang, Th. Schleid, Z. Anorg. Allg. Chem. 2005, 631, 1799.

[4] D.-H. Kang, Th. Komm, Th. Schleid, Z. Kristallogr. 2005, Suppl. 22, 157; D.-H. Kang, Th. Schleid, Z. Anorg. Allg. Chem. 2007, 633, 1205.

[5] M. Ben Hamida, C. Warns, M. S. Wickleder, Z. Naturforsch. 2005, 60 b, 1219; D.-H. Kang, Th. Schleid, Z. Anorg. Allg. Chem. 2006, 632, 91.

[6] M. Palazzi, Acta Crystallogr. 1976, B 32, 3175; H. Sommer, R. Hoppe, Z. Anorg. Allg. Chem. 1977, 430, 199; D.-Y. Seung, P. Gravereau, L. Trut, A. Levasseur, Acta Crystallogr. 1998, C 54,900 .

[7] M. Palazzi, S. Jaulmes, Acta Crystallogr. 1977, B 33, 908; J. E. Iglesias, F. J. Zuniga, W. Nowacki, Z. Kristallogr. 1977, 146, 43; T. K. Bera, J.-H. Song, A. J. Freeman, J. I. Jang, J. B. Ketterson, M. G. Kanatzidis, Angew. Chem. Int. Ed. 2008, 47, 7828.

[8] W. S. Sheldrick, J. Kaub, Z. Naturforsch. 1985, 40 b, 571.

[9] M. Palazzi, S. Jaulmes, P. Laruelle, Acta Crystallogr. 1974, B 30, 2378; A. Pfitzner, T. Bernert, Z. Kristallogr. 2004, 219, 20.

[10] J. Rosenstingl, Österr. Akad. Wiss., Math.-Nat. Klasse, Sitzungsber. 1993, 130, 27; I. J. Fritz, T. J. Isaacs, M. Gottlieb, B. Morosin, Solid State Commun. 1978, 27, 535.

[11] J. E. Jerome, P. T. Wood, W. T. Pennington, J. W. Kolis, Inorg. Chem. 1994, 33, 1733.

[12] G. L. Schimek, J. W. Kolis, Acta Crystallogr. 1997, C 53, 991.

[13] P. T. Wood, G. L. Schimek, J. W. Kolis, Chem. Mater. 1996, 8, 721.

[14] W. Tremel, S. Löken, Eur. J. Inor. Chem. 1998, 1998, 283.

[15] R. G. Lyer, M. G. Kanatzidis, Inorg. Chem. 2002, 41, 3605.

[16] R. G. Lyer, J. Do, M. G. Kanatzidis, Inorg. Chem. 2003, 42, 1475.

[17] R. G. Lyer, M. G. Kanatzidis, Inorg. Chem. 2004, 43, 3656. 
[18] T. K. Bera, R. G. Lyer, C. D. Malliakas, M. G. Kanatzidis, Inorg. Chem. 2007, 46, 8466.

[19] Y. Wu, C. Näther, W. Bensch, Inorg. Chem. 2006, 45, 8835.

[20] D.-H. Kang, Th. Schleid, Z. Anorg. Allg. Chem. 2008, 634, 2050.

[21] D.-H. Kang, Th. Schleid, Z. Kristallogr. 2008, Suppl. 28, 149.

[22] D.-H. Kang, S. Strobel, Th. Schleid, noch unveröffentlicht; D.-H. Kang, Dissertation, Univ. Stuttgart 2009 (in Vorbereitung).

[23] N. Morimoto, Mineral. J. 1954, 1, 160; D. J. E. Mullen, W. Nowacki, Z. Kristallogr. 1972, 136, 48.

[24] Th. Schleid, F. Lissner, Z. Naturforsch. 1996, 51 b, 733.

[25] Th. Schleid, F. Lissner, J. Less-Common Met. 1991, 175, 309.

[26] R. Hoppe, Z. Kristallogr. 1979, 150, 23.

[27] F. Lissner, Th. Schleid, Z. Anorg. Allg. Chem. 1999, 625, 1507.

[28] F. Lissner, Th. Schleid, Z. Anorg. Allg. Chem. 2001, 627, 507.

[29] P. N. Rysanek, A. Mazurier, P. Laruelle, C. Dagron, Acta Crystallogr. 1980, B 36, 2930.

[30] F. Lissner, Th. Schleid, Z. Anorg. Allg. Chem. 1998, 624, 1903.

[31] F. Lissner, Th. Schleid, Z. Anorg. Allg. Chem. 1998, 624, 452.

[32] N. Savigny, C. Adolphe, A. Zalkin, D. H. Templeton, Acta Crystallogr. 1973, B 29, 1532.

[33] G. Kleeff, H. Schilder, H. Lueken, 1995, 621, 963; B. Blaschkowski, Th. Schleid, Z. Anorg. Allg. Chem. 2006, 632, 2149.

[34] G. Sfez, C. Adolphe, Bull. Soc. Franç. Minéral. Crist. 1973, 96, 37.

[35] M. Palazzi, S. Jaulmes, Acta Crystallogr. 1981, B 37, 1340.

[36] R. Hoppe, Angew. Chem. 1966, 78, 52; 1970, 82, 7; 1980, 92, 106; Angew. Chem. Int. Ed. Engl. 1966, 5,$95 ; \mathbf{1 9 7 0}, 9,25 ; \mathbf{1 9 8 0}, 19,110$.

[37] R. Hoppe, Izv. Jugoslav. Centr. Krist. [Zagreb] 1973, 8, 21; in: Crystal Structure and Chemical Bonding in Inorganic Chemistry, C. J. M. Rooymans, A. Rabenau (Eds.), Amsterdam 1975.

[38] P. Lauxmann, S. Strobel, Th. Schleid, Z. Anorg. Allg. Chem. 2002, 628, 2403.

[39] W. Herrendorf, H. Bärnighausen, HABITUS: Programm zur Optimierung der Kristallgestalt für die numerische Absorptionskorrektur als Version X-SHAPE (Version 1.06, Fa. Stoe, Darmstadt 1999), Karlsruhe 1993, Gießen 1996. 
[40] G. M. Sheldrick, SHELX-97: Programmpaket zur Bestimmung und Verfeinerung von Kristallstrukturen aus Beugungsdaten, Göttingen 1997.

[41] A. J. C. Wilson (Ed.), International Tables for Crystallography, Vol. C, $2^{\text {nd }}$ Edit., Kluwer Academic Publishers, Boston, Dordrecht, London 1992. 


\title{
Legenden zu den Abbildungen
}

Abb. 1 Das isolierte, $\psi^{1}$-tetraedrische $\left[\mathrm{AsS}_{3}\right]^{3-}$-Anion und dessen kationische Umgebung aus sechs $\mathrm{Pr}^{3+}$-Kationen in $\operatorname{Pr}_{4} \mathrm{~S}_{3}\left[\mathrm{AsS}_{3}\right]_{2}$

Abb. 2 Koordinationsumgebung der $(\operatorname{Pr} 1)^{3+}-($ oben $)$ und der $(\operatorname{Pr} 2)^{3+}$-Kationen (unten) in der Kristallstruktur von $\mathrm{Pr}_{4} \mathrm{~S}_{3}\left[\mathrm{AsS}_{3}\right]_{2}$

Abb. 3 Die Sulfid-Anionenumgebung der $\left[(\mathrm{S} 1)(\operatorname{Pr} 1)_{4}\right]^{10+}-$ Tetrader $($ oben $)$ und der $\left[(\mathrm{S} 2)(\operatorname{Pr} 2)_{4}\right]^{10+}-$ Tetraeder (unten) in der Kristallstruktur von $\operatorname{Pr}_{4} \mathrm{~S}_{3}\left[\mathrm{AsS}_{3}\right]_{2}$

\begin{abstract}
Abb. $4{ }_{\infty}^{1}\left\{\left[(\mathrm{~S} 1)(\operatorname{Pr} 1)_{4 / 2}\right]^{4+}\right\}$-Kette aus trans-kantenverknüpften $\left[\mathrm{SPr}_{4}\right]^{10+}$-Tetraedern entlang [001] (oben) und deren Anordnung nach Art einer hexagonalen Stabpackung (unten) in der Kristallstruktur von $\operatorname{Pr}_{4} \mathrm{~S}_{3}\left[\mathrm{AsS}_{3}\right]_{2}$
\end{abstract}

Abb. $5{ }_{\infty}^{2}\left\{\left[(\mathrm{~S} 2)(\operatorname{Pr} 2)_{4 / 4}\right]^{+}\right\}-$Schicht aus über vier Kanten kondensierten $\left[\mathrm{SPr}_{4}\right]^{10+}-$ Tetraedern in der Kristallstruktur von $\operatorname{Pr}_{4} \mathrm{~S}_{3}\left[\mathrm{AsS}_{3}\right]_{2}$ parallel (100)

\begin{abstract}
Abb. 6 Blick auf die gesamte Kristallstruktur des Praseodym(III)-Sulfid-Thioarsenats(III) $\mathrm{Pr}_{4} \mathrm{~S}_{3}\left[\mathrm{AsS}_{3}\right]_{2}$ in Richtung der $c$-Achse, der zeigt, dass sich die lone pairs an den $\mathrm{As}^{3+}$-Kationen der $\left[\mathrm{AsS}_{3}\right]^{3-}$-Einheiten zu den ${ }_{\infty}^{1}\left\{\left[(\mathrm{~S} 1)(\operatorname{Pr} 1)_{4 / 2}\right]^{4+}\right\}-$ Ketten hin ausrichten
\end{abstract}

\begin{abstract}
Abb. 7 Projektion der Kristallstruktur des Lanthan(III)-Oxid-Thioarsenats(III) $\mathrm{La}_{4} \mathrm{O}_{3}\left[\mathrm{AsS}_{3}\right]_{2}$ auf (001) unter Hervorhebung der ${ }_{\infty}^{1}\left\{\left[\mathrm{O}_{3} \mathrm{La}_{4}\right]^{6+}\right\}$-Dreifachketten aus kantenverknüpften $\left[\mathrm{OLa}_{4}\right]^{10+}$ Tetraedern (oben) und die Kristallstruktur von $\mathrm{Nd}_{4} \mathrm{~S}_{3} \mathrm{Br}_{6}$ mit schwefelanalogen Dreifachketten ${ }_{\infty}^{1}\left\{\left[\mathrm{~S}_{3} \mathrm{Nd}_{4}\right]^{6+}\right\}$ entlang der $b$-Achse (unten)
\end{abstract}




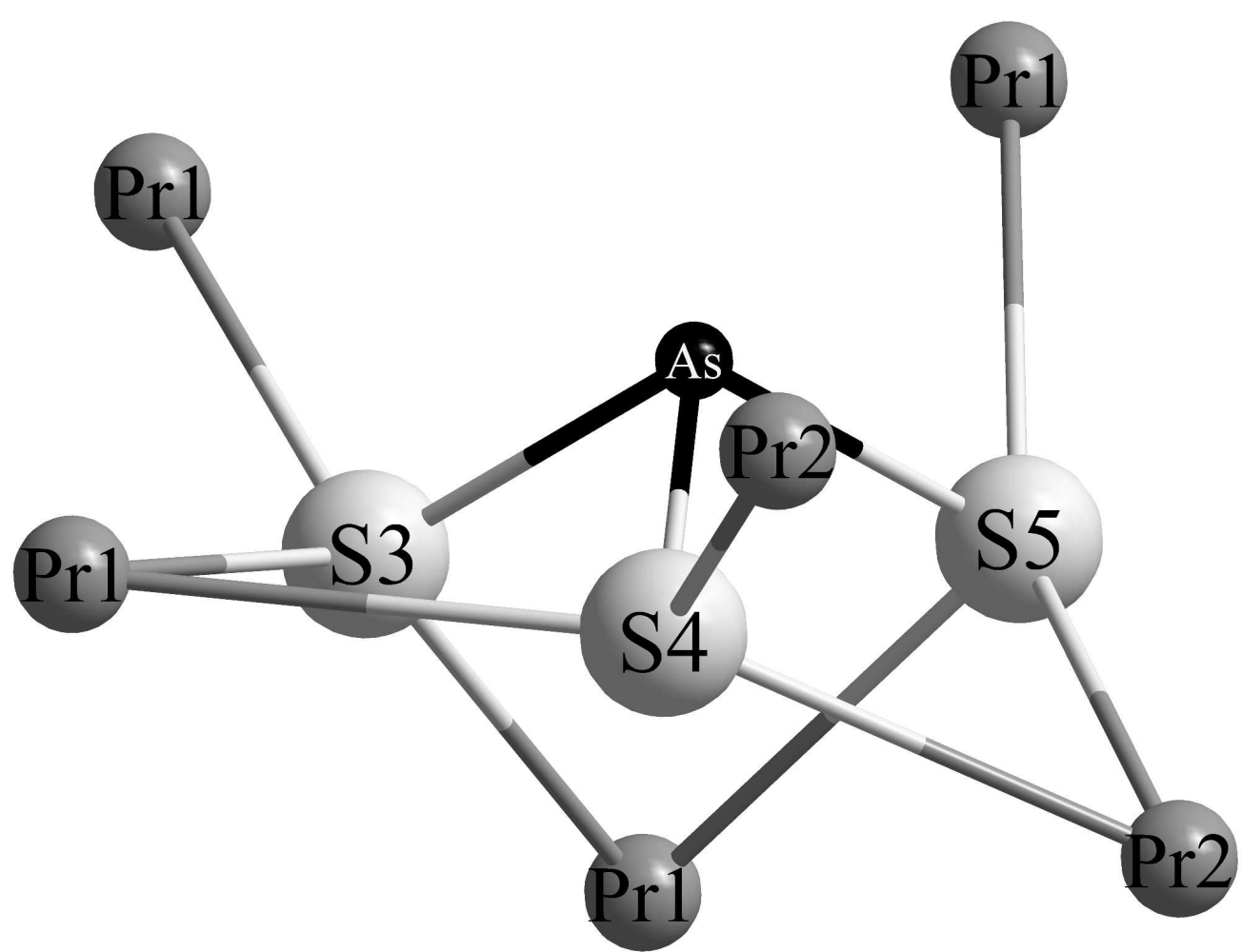

Abb. 1 Das isolierte, $\psi 1$-tetraedrische [AsS3]3--Anion und dessen kationische Umgebung aus sechs $\mathrm{Pr} 3+-$ Kationen in $\mathrm{Pr} 4 \mathrm{~S} 3[\mathrm{AsS} 3] 2$ $170 \times 129 \mathrm{~mm}(600 \times 600 \mathrm{DPI})$ 

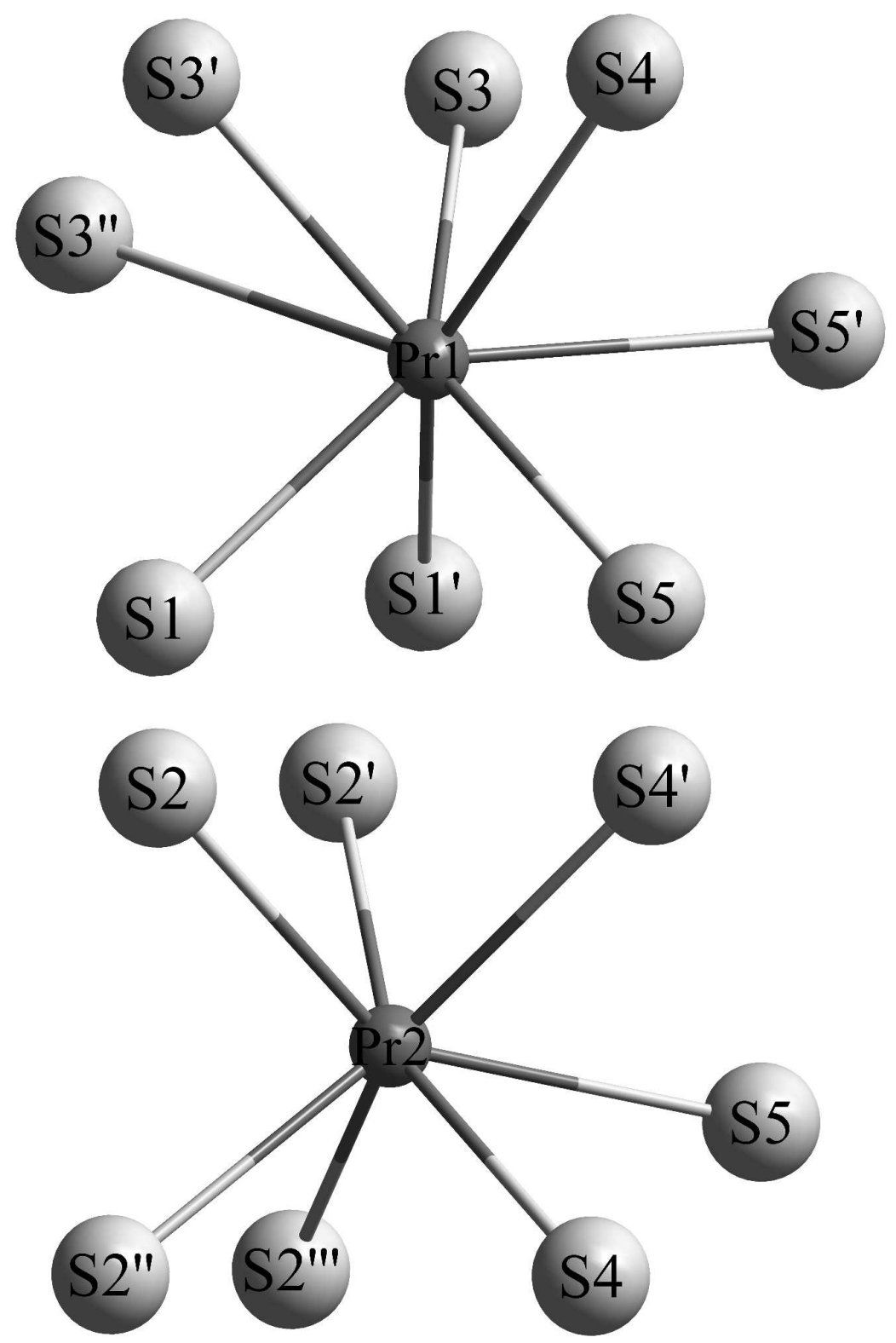

Abb. 2 Koordinationspolyeder aus Schwefelatomen um die (Pr1)3+- (oben) und die (Pr2)3+Kationen (unten) in der Kristallstruktur von Pr4S3[AsS3]2

$$
170 \times 256 \mathrm{~mm}(600 \times 600 \mathrm{DPI})
$$



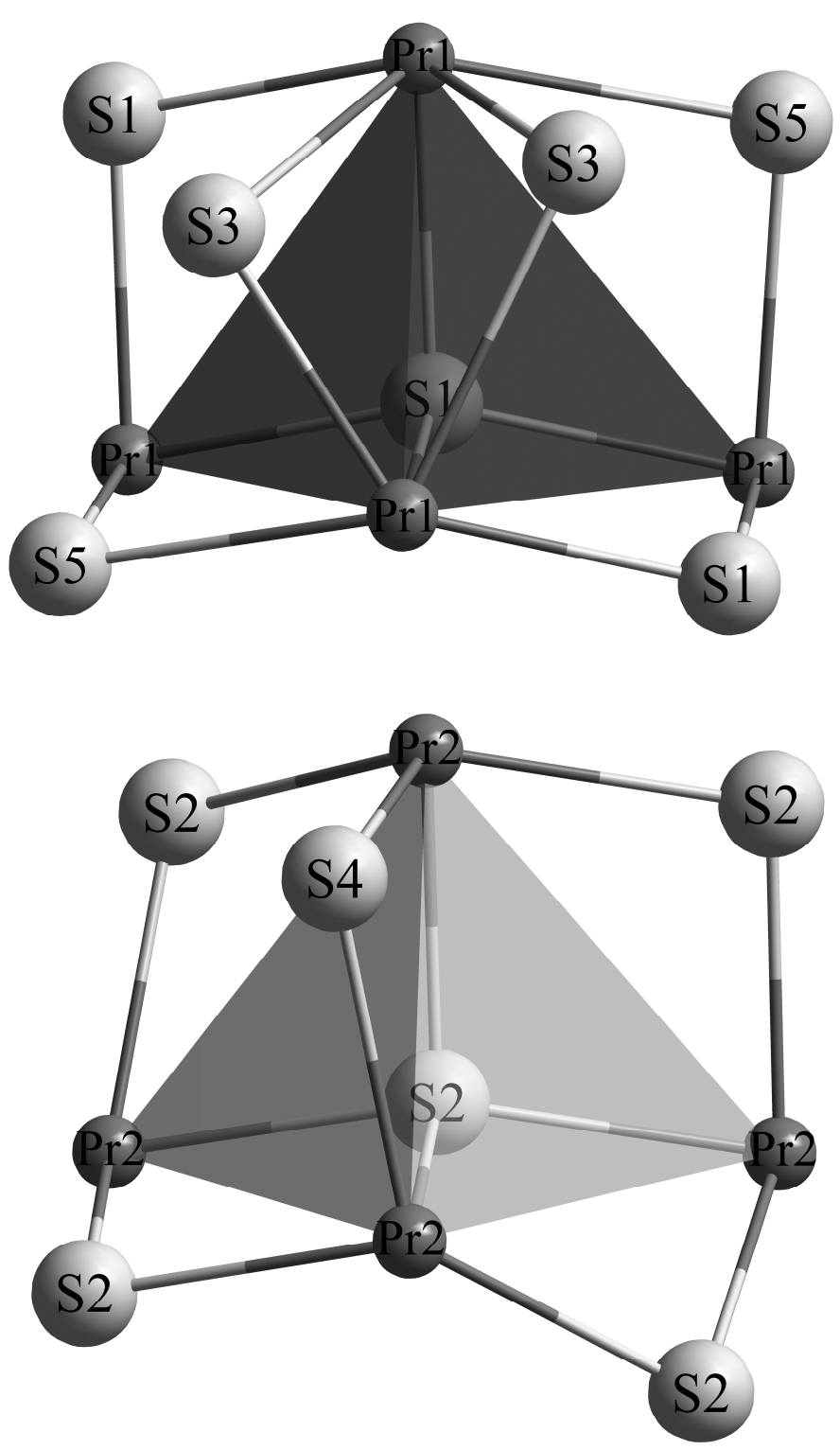

Die Sulfid-Anionenumgebung der [(S1)(Pr1)4]10+-Tetrader (oben) und der [(S2)(Pr2)4]10+Tetraeder (unten) in der Kristallstruktur von Pr4S3[AsS3]2 $170 \times 280 \mathrm{~mm}(600 \times 600 \mathrm{DPI})$ 


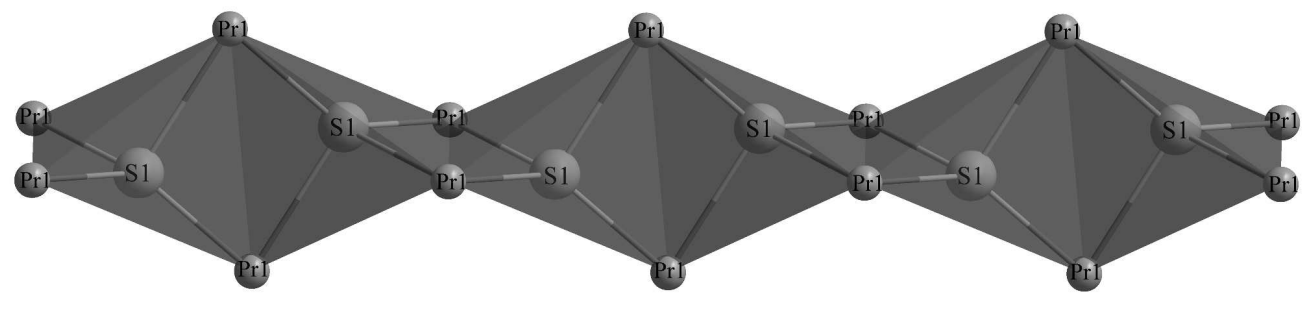

[001]

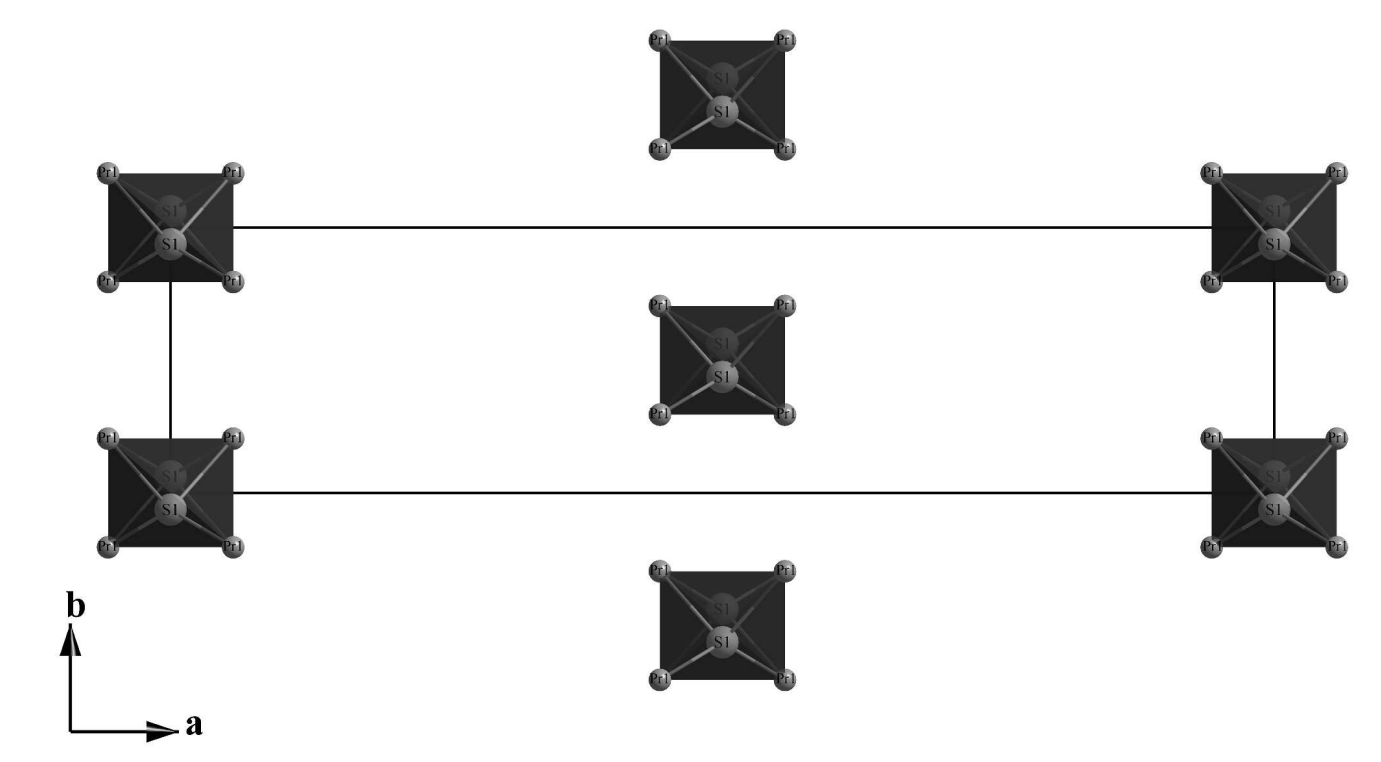

$\{[(\mathrm{S} 1)(\operatorname{Pr} 1) 4 / 2] 4+\}-K e t t e$ aus trans-kantenverknüpften [SPr4]10+-Tetraedern entlang [001] (oben) und deren Anordnung nach Art einer hexagonalen Stabpackung (unten) in der Kristallstruktur von Pr4S3[AsS3]2 $170 \times 156 \mathrm{~mm}(600 \times 600 \mathrm{DPI})$ 


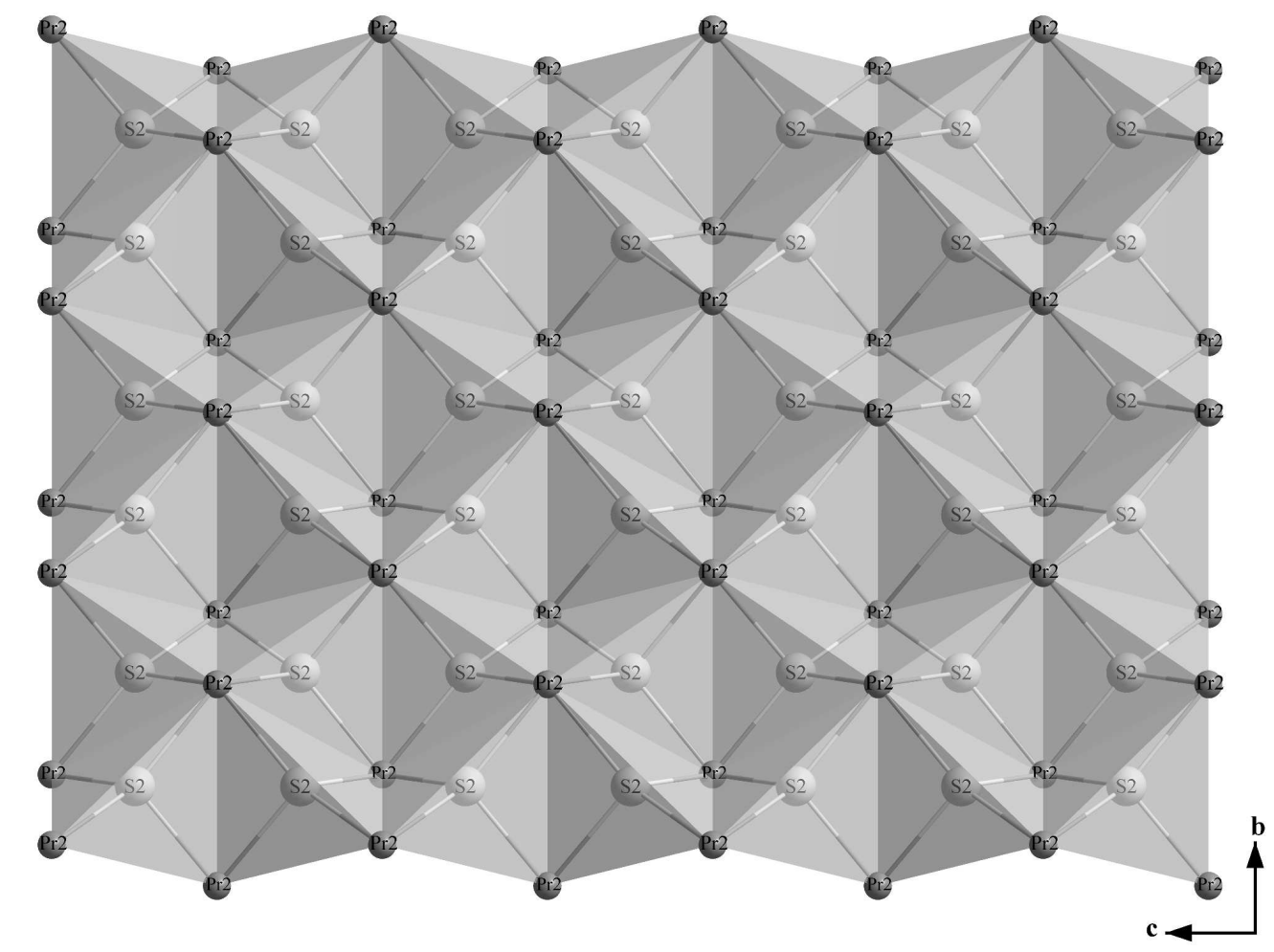

$\{[(\mathrm{S} 2)(\operatorname{Pr} 2) 4 / 4]+\}$-Schicht aus über vier Kanten kondensierten [SPr4]10+-Tetraedern in der Kristallstruktur von Pr4S3[AsS3]2 parallel (100) $170 \times 129 \mathrm{~mm}(600 \times 600 \mathrm{DPI})$ 


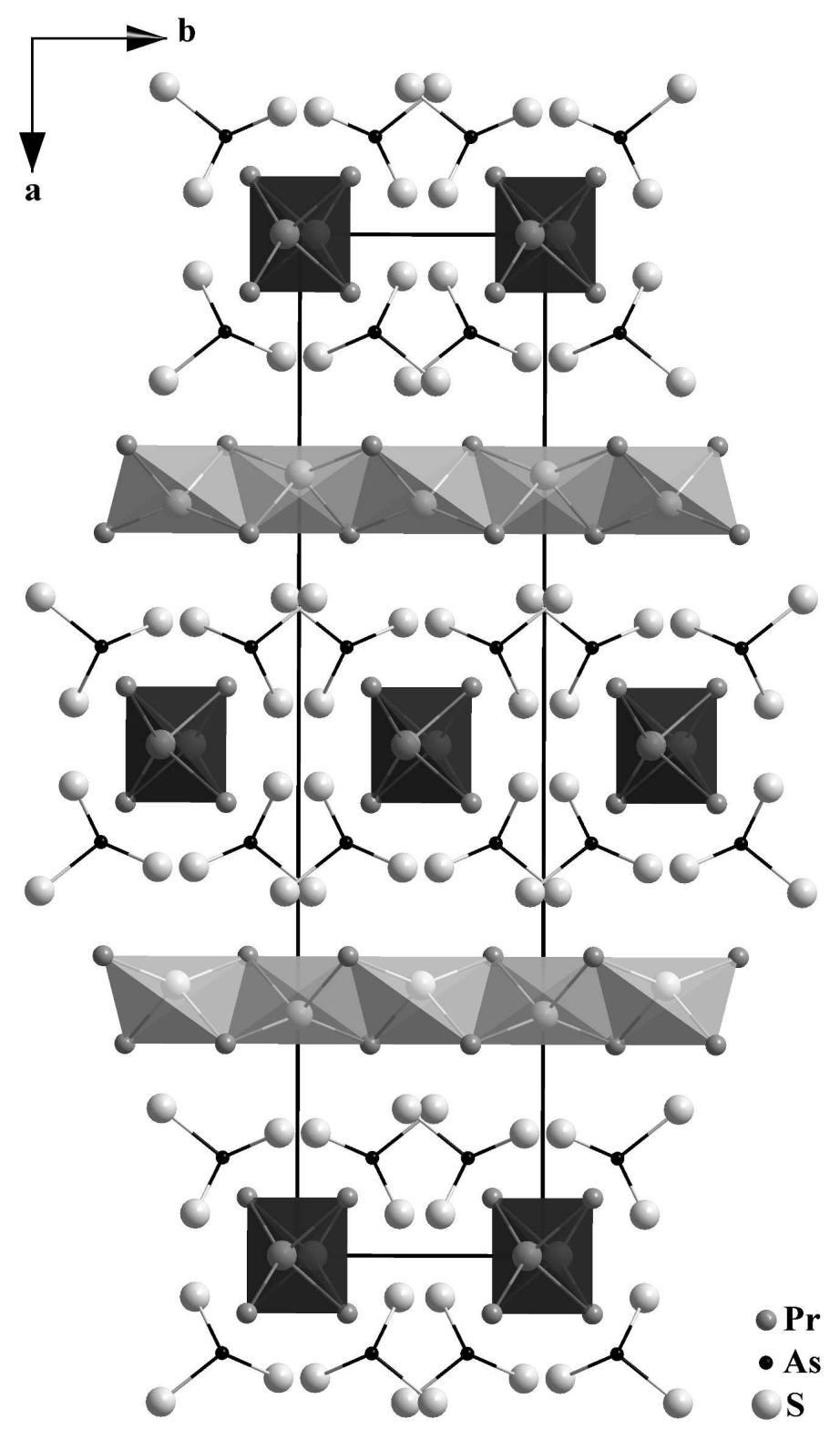

Blick auf die gesamte Kristallstruktur des Praseodym(III)-Sulfid-Thioarsenats(III) Pr4S3[AsS3]2 in Richtung der c-Achse, der zeigt, dass sich die lone pairs an den As3+-Kationen der [AsS3]3-Einheiten zu den $\{[(\mathrm{S} 1)(\operatorname{Pr} 1) 4 / 2] 4+\}$-Ketten hin ausrichten $170 \times 296 \mathrm{~mm}(600 \times 600 \mathrm{DPI})$ 

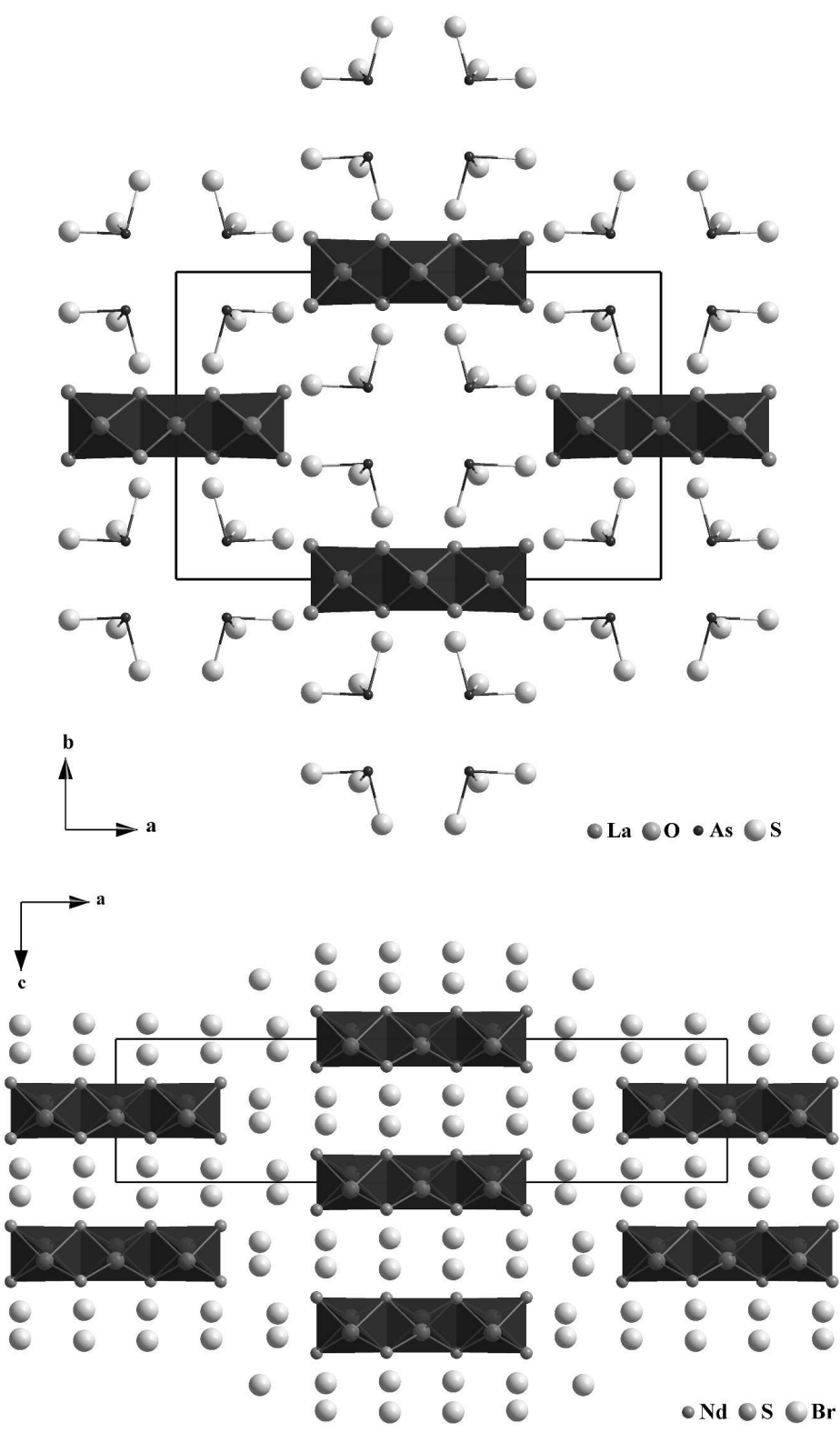

Projektion der Kristallstruktur des Lanthan(III)-Oxid-Thioarsenats(III) La4O3[AsS3]2 auf (001) unter Hervorhebung der \{[O3La4]6+\}-Dreifachketten aus kantenverknüpften [OLa4]10+Tetraedern (oben) und die Kristallstruktur von Nd4S3Br6 mit schwefelanalogen Dreifachketten $\{[\mathrm{S} 3 \mathrm{Nd} 4] 6+\}$ entlang der b-Achse (unten) $170 \times 285 \mathrm{~mm}(600 \times 600 \mathrm{DPI})$ 